\title{
Distribución espacio-temporal de variables fisicoquímicas y biológicas en el hábitat del tiburón ballena Rhincodon typus (Orectolobiformes: Rhincodontidae) al norte del Caribe Mexicano
}

\author{
Natalí Cárdenas-Palomo ${ }^{1}$, Jorge Herrera-Silveira ${ }^{2} \&$ Óscar Reyes ${ }^{2}$ \\ 1. Pronatura Península de Yucatán, A.C. Calle 32 No. 269 x 47 y 47 A Col. Pinzón II C.P. 97207 Mérida, Yucatán. \\ México; natalicp@pronatura-ppy.org.mx, natali_cp@hotmail.com \\ 2. Centro de Investigación y Estudios Avanzados del IPN. Carr. Antigua a Progreso km.6 Mérida, Yuc. 97310, México; \\ jherrera@mda.cinvestav.mx, freyes@mda.cinvestav.mx
}

Recibido 04-V-2009. Corregido 20-IX-2009. Aceptado 21-X-2009.

\begin{abstract}
Spatial and temporal distribution of physicochemical features in the habitat of whale shark Rhincodon typus (Orectolobiformes: Rhincodontidae) in the north of Mexican Caribbean. Large groups of whale sharks (Rhincodon typus) are common in the North Mexican Caribbean and gather between May and September each year. We describe their spatial and temporal distribution, and the physicochemical (temperature, dissolved oxygen, light extinction coefficient and salinity) and biological conditions during the presence and absence seasons of R. typus. A total of 26 sampling stations were monitored to record whale shark sightings and physicochemical variables during 10 field campaigns from April 2005 to March 2006. At each station, zooplankton and water samples, for chlorophyll-a and nutrients determination, were collected. Physicochemical conditions were significantly different between presence-absence seasons (ANOSIM, Rglobal =0.632). The R. typus season was characterized by low salinity values, and higher temperature, chlorophyll-a, dissolved oxygen and inorganic nitrogen concentrations values. Average zooplankton biomass was lower during the absence season, while recorded the maximum values during the presence one. Furthermore, these values were also observed in areas with higher species abundances, supporting the hypothesis that the site is used by $R$. typus primarily as a foraging area. We conclude that physicochemical conditions of the study area promote the biological productivity, which explains the spatial and temporal variability of R. typus. Rev. Biol. Trop. 58 (1): 399-412. Epub 2010 March 01.
\end{abstract}

Key words: Rhincodon typus, upwelling, habitat, spatial pattern distribution, Mexican Caribbean.

El tiburón ballena Rhincodon typus (Smith 1828), el pez más grande del mundo, alcanza una longitud entre 16 y $18 \mathrm{~m}$ y hasta 34 toneladas de peso. Se distribuye entre $\operatorname{los} 30^{\circ} \mathrm{N} \mathrm{y}$ $35^{\circ} \mathrm{S}$, abarcando todos los mares templados y cálidos del mundo, encontrándose en ambientes oceánicos y costeros (Compagno 1984, Colman 1997, CITES 2000, Eckert \& Stewart 2001). Es una especie filtradora que se alimenta principalmente de zooplancton y pequeños peces (Gudger 1941, Compagno 1984, Colman 1997, Clark \& Nelson 1997). A pesar de su amplia distribución, son escasos los sitios en el mundo donde la presencia $R$. typus es predecible y permanente (CITES 2000); probablemente, ésta sea una de las causas por la que la especie ha sido pobremente estudiada a nivel mundial. El interés socioeconómico y científico por el tiburón ballena ha incrementado en las dos últimas décadas debido a que es el centro de una industria ecoturística altamente lucrativa en sitios dónde se congrega de manera estacional (Cárdenas-Torres et al. 2007; Quiros 2007).

La información acerca de las condiciones del hábitat que hacen posible la presencia del 
tiburón ballena es escasa. En sitios como el Arrecife Ningaloo (Australia), Gladden Spit (Belice) y Baja California (México), se han realizado estudios con este objetivo (Heyman et al. 2001; Wilson et al. 2001, Ketchum 2003, Graham et al. 2007), sin embargo aún existen vacíos de información en otros sitios de agregación de $R$. typus. La caracterización del hábitat de una especie permite conocer la forma en la cual se comportan las variables ambientales en tiempo y espacio, así como reconocer la heterogeneidad ambiental e identificar posibles amenazas, lo cual constituye información esencial para el diseño de estrategias adecuadas de manejo y conservación.

De manera general, se considera que la distribución y abundancia del tiburón ballena se encuentra influenciada por procesos oceanográficos tales como surgencias, corrientes costeras y frentes, que incrementan la productividad en el ambiente marino favoreciendo las concentraciones localizadas de alimento (Colman 1997, Taylor \& Pearce 1999, Norman 2000, Eckert \& Stewart 2001, Wilson et al. 2001, Duffy 2002, Ketchum 2003).

El área marina ubicada al norte de la Península de Yucatán (sureste de México), se caracteriza por su alta productividad biológica como resultado de un sistema de surgencia de aguas profundas que penetran el canal de Yucatán avanzando hasta el Golfo de México (Merino 1992, Pérez de los Reyes et al. 1996). Durante el periodo de mayo a septiembre de cada año, cardúmenes de tiburón ballena se observan en esta zona, posiblemente atraídos por la abundancia de alimento. La necesidad de generación de información para la conservación de la especie en esta zona se ha incrementado desde 2003 debido a que en las comunidades locales los viajes de observación y nado libre con esta especie se han convertido en la actividad turística más lucrativa (Solares 2005). Sin embargo, existen grandes vacíos de información científica acerca de la ecología del tiburón ballena y su hábitat en esta zona del Caribe, sitio que se ha sido reconocido como uno de los más importantes para la especie registrándose grupos de más de cien organismos y más de 300 avistamientos por temporada (SEMARNAT-CONANP 2005, Reyes-Mendoza \& Cárdenas-Palomo 2006). Por esta razón, el conocimiento de las condiciones que determinan la distribución del tiburón ballena y la caracterización de su hábitat son de vital importancia para la conservación de la especie a escala local y mundial.

El presente trabajo pretende aportar información básica sobre la caracterización ecológica del hábitat del tiburón ballena al norte del Caribe mexicano, con el objetivo de brindar herramientas para el diseño de estrategias de manejo y conservación de la especie a corto, mediano y largo plazo, determinando variaciones temporales y espaciales en avistamientos de tiburón ballena y su relación con las condiciones fisicoquímicas y biológicas del área marina ubicada al norte del Caribe mexicano.

\section{MATERIALES Y MÉTODOS}

Área de estudio: El área de estudio comprende la región marina ubicada al norte del Caribe mexicano y sureste del Golfo de México (Fig. 1), la cual constituye una zona de transición entre el Golfo de México y el Mar Caribe. El clima es cálido semiárido a subhúmedo con lluvias en verano; la temperatura media anual es de $22-26^{\circ} \mathrm{C}$ (Arriaga-Cabrera et al. 1998). La zona se caracteriza por tres estaciones climáticas: seca (marzo a mayo) caracterizada por baja precipitación $(0-50 \mathrm{~mm})$; lluvias (junio-octubre) con alta precipitación $(>500 \mathrm{~mm})$ y la época de nortes y frentes fríos (noviembre-febrero) con precipitación moderada $(20-50 \mathrm{~mm})$, vientos fuertes $(50-90 \mathrm{~km} / \mathrm{h})$ y bajas temperaturas $\left(<22^{\circ} \mathrm{C}\right)$ (Herrera-Silveira et al. 1998).

La región es considerada de alta productividad biológica a consecuencia del fenómeno de surgencia ("surgencia de Yucatán"), la cual permanece todo el año (carácter cuasipermanente) mostrando diferencias temporales ya que intensifica su fuerza durante primavera y verano (Ruíz-Renteria \& Merino 1989, Merino 1992, Pérez de los Reyes et al. 1996, Cereida-Estrada et al. 1998). Se han descrito 


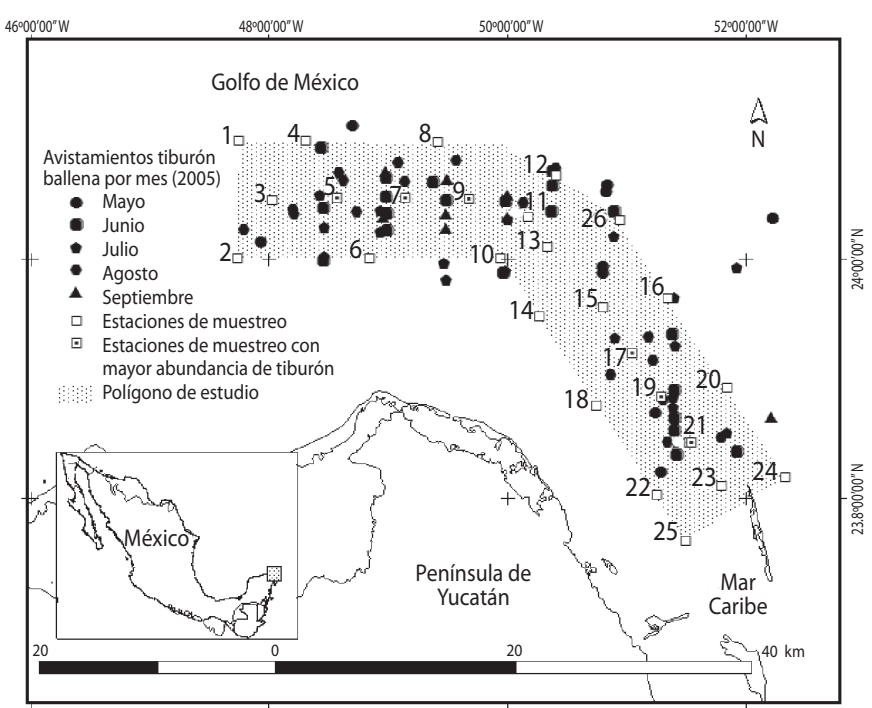

Fig. 1. Área de estudio y avistamientos de tiburón ballena registrados durante mayo a septiembre de 2005. La mayoría de los datos de avistamientos fueron colectados mediante vuelos realizados por la CONANP (DOMINO 2005).

Fig 1. Study area and sightings of whale shark during May to September 2005. Most sightings are collected by CONANP (arial surveys) (DOMINO 2005).

dos posibles mecanismos del origen de esta surgencia que no se encuentran relacionados con la divergencia ocasionada por el viento tal como sucede en las grandes surgencias mundiales: la fricción de la corriente de Yucatán al chocar contra la cuesta del borde este de la Plataforma de Yucatán (Cochrane 1966) y la interacción entre la corriente de Yucatán y una contracorriente que corre por debajo hacia el sur (García 1990).

Programa de muestreo y análisis de laboratorio: Con base en el mapa de avistamientos de tiburón ballena de la temporada 2004 elaborado por la Comisión Nacional de Áreas Naturales Protegidas (CONANP) a través de su Proyecto "DOMINO", se definió el polígono de estudio abarcando las zonas donde se registra la presencia de tiburón ballena. Dentro del polígono que comprende un área de $536.3 \mathrm{~km}^{2}$, se estableció una red de 26 estaciones distribuidas de manera homogénea (Fig. 1). Durante el periodo abril de 2005 a marzo de 2006, se realizaron salidas de campo con una periodicidad mensual a fin de registrar avistamientos de tiburón ballena y evaluar las condiciones fisicoquímicas [temperatura superficial (TSM), salinidad, oxígeno disuelto] utilizando un YSI 660 y biológicas (concentración de cl- $a$ y biomasa zooplanctónica). Con la ayuda de un irradiómetro se midió el coeficiente de extinción de luz (k1) y los nutrientes (nitrógeno inorgánico disuelto -suma de concentración de nitrato, nitrito y amonio-, silicatos y fosfatos) y clorofila- $a$ fueron determinados a partir de una muestra de agua colectada en cada estación a nivel superficial las cuales fueron conservadas a baja temperatura $\left(4^{\circ} \mathrm{C}\right)$ hasta el momento de su análisis. En cada estación de muestreo se realizó un arrastre horizontal de zooplancton a nivel superficial, utilizando una red de luz de malla de $300 \mu \mathrm{m}$ y con un flujómetro Ocean Instrument calibrado de acuerdo con las recomendaciones de la CICAR (UNESCO 1979). Las muestras fueron fijadas en el campo utilizando formaldehído en agua de mar neutralizado con borato de sodio a un $\mathrm{pH}=7.5-8$. Durante los meses de octubre de 2005 y enero 
de 2006, no fue posible realizar el trabajo de campo debido al paso del huracán Wilma y la presencia de nortes respectivamente.

En el laboratorio, las determinaciones de nutrientes se realizaron siguiendo los métodos descritos por Strickland \& Parsons (1972) y la cuantificación de clorofila- $a$ se llevó a cabo a través de la técnica de acetona al $90 \%$ y posteriormente analizando espectrofotométricamente el extracto de acuerdo a la fórmula propuesta por Jefrey y Humphrey en 1975, las cuales son descritas en Parsons et al. (1984). La biomasa zooplanctónica fue cuantificada por la técnica de peso húmedo, expresándose en $\mathrm{g} / 1000 \mathrm{~m}^{3}$ (Omori \& Ikeda 1984).

En cada muestreo fueron registrados los avistamientos de tiburón ballena (coordenadas, hora, día, número de organismos), los cuales se complementaron con los datos colectados por la CONANP mediante cinco sobrevuelos aéreos realizados entre mayo y septiembre de 2005. Las fechas de los censos aéreos no tuvieron más de cinco días de diferencia con los días en los que se realizó la colecta de datos fisicoquímicos. La ruta de los censos aéreos fue realizada en transectos paralelos, los cuales cubrieron en su totalidad el polígono de estudio (DOMINO 2005).

Análisis de datos: Las coordenadas geográficas de cada avistamiento de tiburón ballena (tanto de avistamientos registrados desde la embarcación marina, como en sobrevuelos) fueron incorporadas a un mapa utilizando el programa ArcView Versión 3.1. Cada avistamiento fue asociado a la estación de muestreo más cercana. Los datos hidrobiológicos fueron agrupados en dos temporadas para su análisis: presencia (mayo a septiembre de 2005) y ausencia de tiburón ballena (abril, noviembre, diciembre de 2005; febrero y marzo de 2006).

Los datos de cada estación de muestreo de los meses correspondientes a cada temporada fueron promediados; se realizó una prueba de Kruskal-Wallis debido a que los datos no se ajustan a una distribución normal (la representación grafica del análisis se hizo en diagramas de cajas y bigotes), utilizando los programas STATGRAPHICS Plus 4.1 e INFOSTAT Versión 1.1. Para determinar si las condiciones ambientales correspondientes a los meses de la misma temporada (presencia y ausencia) eran similares, se aplicó el análisis de escalamiento multidimensional noparamétrico (MDS). Se realizó un análisis de similitudes con 126 permutaciones (Analysis of Similarities, ANOSIM) para determinar si el agrupamiento por temporada era significativo y, a través de la prueba SIMPER (Similarity Percentages) (Clarke \& Warwick 1994), fueron identificadas las variables que caracterizaban y discriminaban las temporadas, utilizando el Programa PRIMER 6.1 (Plymouth Routines In Multivariate Ecological Research).

\section{RESULTADOS}

\section{Avistamientos de tiburón ballena:} Durante los recorridos para la toma de datos fisicoquímicos y biológicos, se registró un total de 16 avistamientos de tiburón ballena, contabilizándose un total de 52 organismos. En los sobrevuelos realizados por la CONANP se registró un total de 43 avistamientos y 258 organismos (Fig. 1). La estación de muestreo con mayor número de avistamientos totales (96) y organismos contabilizados (192) fue la estación 2 (Fig. 2).

Variables Hidrológicas: La TSM durante la temporada de presencia de tiburón ballena $\left(27.8^{\circ} \mathrm{C}\right.$ DS 0.58$)$ fue mayor a la registrada en la temporada de ausencia de la especie $\left(25.0^{\circ} \mathrm{C}\right.$ DS 0.42), siendo la diferencia significativa (Kruskal-Wallis, p<0.0001) (Fig. 3a). Los valores de salinidad mostraron diferencias significativas entre temporadas (Kruskal-Wallis, $\mathrm{p}<0.0001$ ) (Fig. 3b). La salinidad promedio en la temporada de tiburón ballena fue menor (35.4 DS 0.11) a la registrada en la temporada sin tiburón (36.7 DS 0.17). El porcentaje de saturación de oxígeno presentó diferencias significativas entre temporadas (Kruskal-Wallis, $\mathrm{p}=0.0009$ ) siendo más bajo en la temporada de ausencia de tiburón (90.3\% DS 7.34). Durante la temporada de presencia de tiburón ballena, 


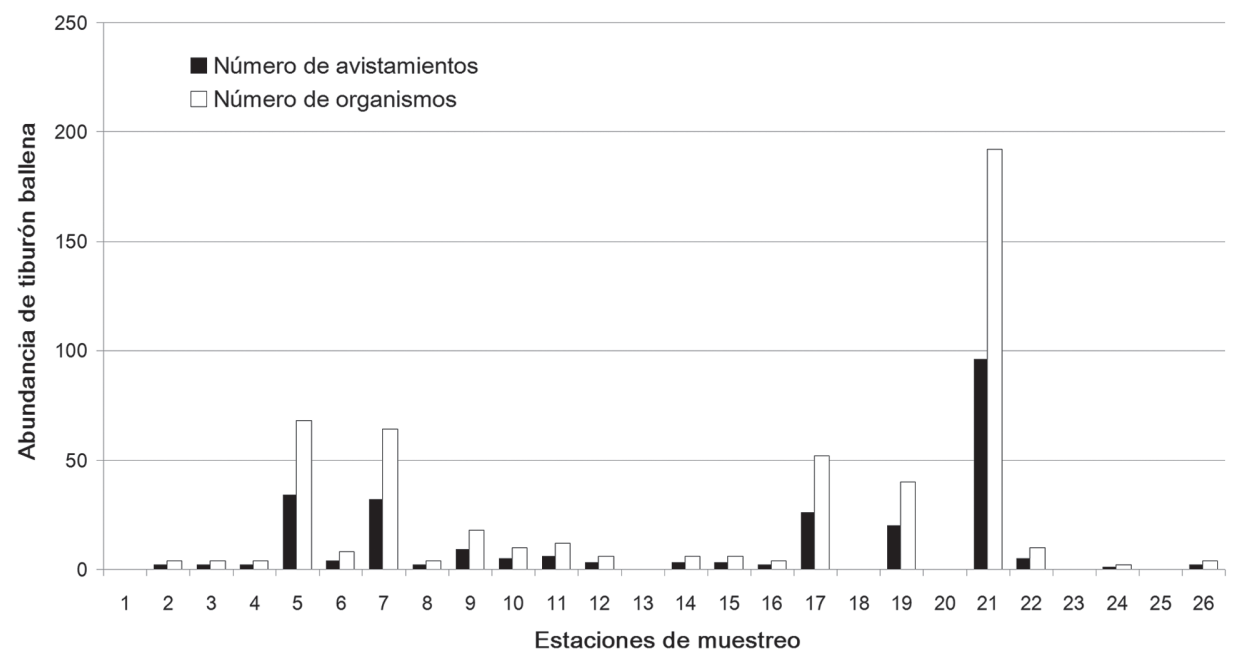

Fig. 2. Número de avistamientos y organismos contabilizados de tiburón ballena en cada estación de muestreo, durante mayo a septiembre de 2005. La mayoría de los datos fueron colectados mediante vuelos realizados por la CONANP (DOMINO 2005).

Fig 2. Number of sightings and individual accounted of whale shark in each sampling point, during May to September 2005 .

se registró mayor variabilidad con relación a esta variable (69.9\% a $129.5 \%$ ) (Fig. 3c). Durante la temporada de presencia del tiburón ballena, el coeficiente de extinción de luz promedio registrado $\left(0.44 \mathrm{~m}^{-1} \mathrm{DS} 0.08\right)$ fue mayor que el observado en temporada de ausencia de la especie $\left(0.27 \mathrm{~m}^{-1}\right.$ DS 0.07) (Fig. 3d). La diferencia en esta variable fue significativa entre temporadas (Kruskal-Wallis, $\mathrm{p}<0.0001$ ).

La concentración de nitrógeno inorgánico disuelto ( $\mathrm{DIN}=\mathrm{NO}_{3}+\mathrm{NO}_{2}+\mathrm{NH}_{4}$ ) presentó diferencias significativas entre temporadas (Kruskal-Wallis, $\mathrm{p}<0.0001$ ), siendo en promedio mayor la concentración en temporada de presencia de tiburón ballena $(6.12 \mu \mathrm{mol} / \mathrm{L}$ DS 1.33) (Fig. 3e). La concentración de fosfatos fue significativamente diferente entre temporadas (Kruskal-Wallis, $\mathrm{p}=0.0001$ ), registrándose concentraciones promedio más altas durante la temporada de ausencia de tiburón ballena (Fig. 3f), mientras que la concentración promedio de silicatos durante la temporada de presencia de tiburón ballena fue ligeramente menor que la observada en la temporada de ausencia, aunque la diferencia entre temporadas no fue significativa en relación con este nutriente (KruskalWallis, $\mathrm{p}=0.8333$ ) (Fig. 3g).

Variables biológicas: La concentración promedio de Clorofila- $a$ durante la temporada de tiburón ballena fue de $1.53 \mathrm{mg} / \mathrm{m}^{3}$ (DS $0.28)$, mientras que en la temporada sin tiburón la concentración fue de $0.83 \mathrm{mg} / \mathrm{m}^{3}$ (DS0.30). La diferencia entre temporadas fue significativa (Kruskal-Wallis, $\mathrm{p}<0.0001$ ), presentando mayor variabilidad durante la temporada de tiburón (Fig. 4a).

La biomasa promedio de zooplancton durante la temporada de tiburón ballena fue de $26.9 \mathrm{~g} / 1000 \mathrm{~m}^{3}$ (DS 21.67), mientras que la registrada durante la temporada sin tiburón fue de $42.8 \mathrm{~g} / 1000 \mathrm{~m}^{3}$ (DS 13.93), sin embargo, el máximo de biomasa zooplanctónica se registró durante la temporada de tiburón ballena $\left(222.4 \mathrm{~g} / 1000 \mathrm{~m}^{3}\right)$ en la estación 2 , sitio donde fue contabilizada la mayor abundancia de 

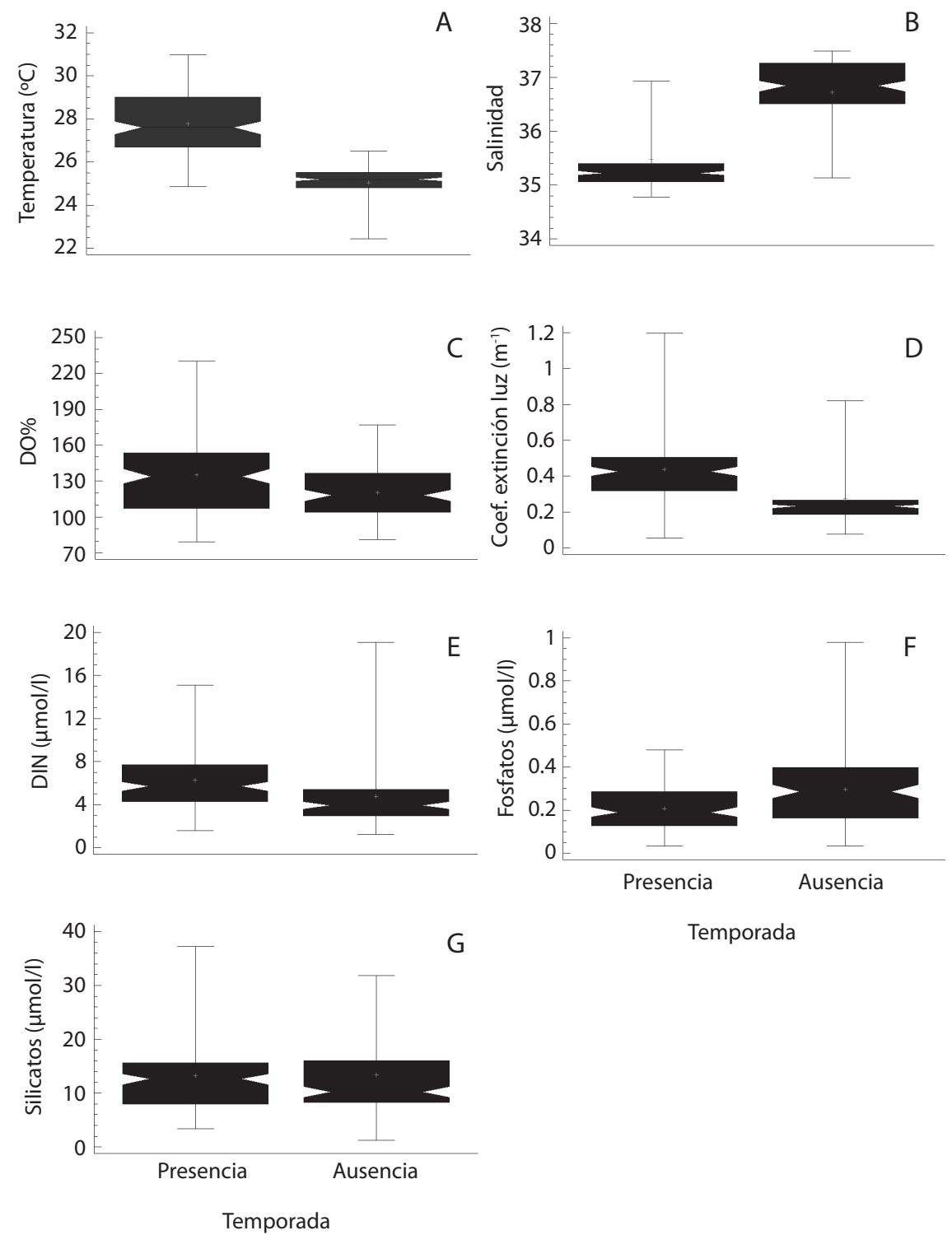

Fig. 3. Diagrama de cajas de las variables hidrológicas para la temporada de ausencia y presencia de tiburón ballena en la zona.

Fig 3. Diagram boxes for hydrological variables for the season absence and presence of whale sharks in the area.

tiburones ballena. Se observó diferencia significativa en esta variable entre ambas temporadas (Kruskal-Wallis, p<0.0001) (Fig. 4b). Con relación a la distribución espacial de biomasa zooplanctónica, se observó en la temporada de presencia del tiburón ballena mayores abundancias zooplanctónicas en las estaciones 4 y 21, ambas con un promedio mayor a $80 \mathrm{~g} / 1000 \mathrm{~m}^{3}$ y en temporada de ausencia de la especie la mayor abundancia de zooplancton se ubicó en las estaciones 1 y 4 con un promedio mayor a $60 \mathrm{~g} / 1000 \mathrm{~m}^{3}$ (Fig. 5). 

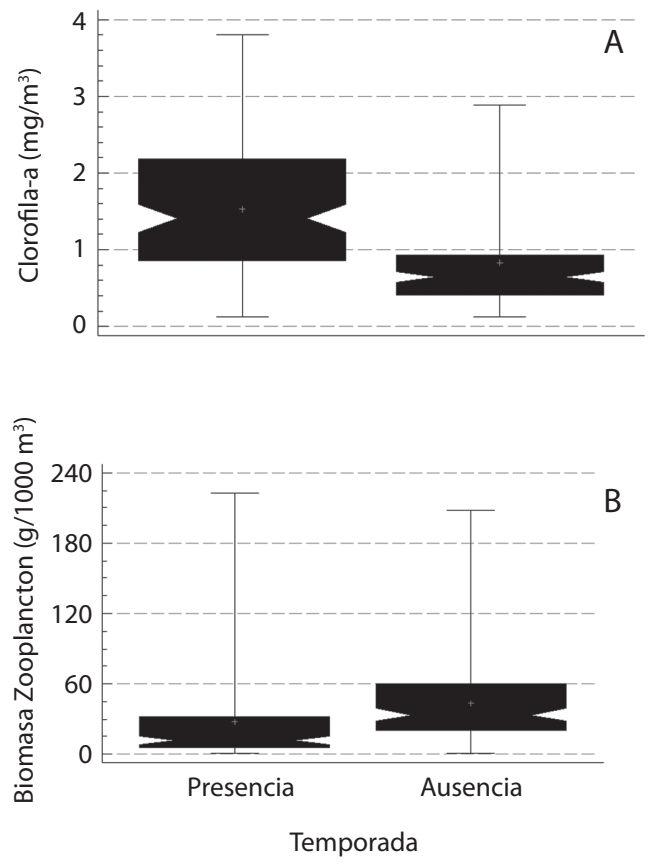

Fig. 4. Diagrama de cajas de las variables biológicas para la temporada de ausencia y presencia de tiburón ballena en la zona. (A.) Concentración de clorofila-a superficial (B.) biomasa zooplanctónica.

Fig 4. Box and Whisker Plot of biological variables for the two seasons: absence and presence of whale sharks. (A.) chlorophyll- $a$ superficial concentration; (B.) zooplankton biomass.

La representación bidimensional del MDS (Fig. 6) mostró una clara separación entre los meses correspondientes a cada una de las temporadas, registrando un valor de "stress" bajo (0.1). La prueba de ANOSIM detectó que la agrupación en temporadas fue significativa $\left(R_{\text {global }}=0.632\right)$, mientras que el análisis SIMPER identificó que el coeficiente de extinción de luz, la temperatura, los fosfatos y la biomasa zooplanctónica, son las variables de mayor importancia en estas diferencias, al contribuir con más del $47 \%$ de la disimilitud (Cuadro 1).

\section{DISCUSIÓN}

Variables hidrológicas: Durante la temporada de presencia de tiburón ballena, la TSM promedio fue mayor que en la temporada de ausencia de la especie (Fig. 3a), debido probablemente a que éstos meses (mayo-septiembre) corresponden a las estaciones climatológicas más cálidas de la región (secas y con lluvias) (Herrera-Silveira et al. 1998). La TSM registrada en cada una de las temporadas, coinciden con reportes anteriores para la zona: de 25 a $27^{\circ} \mathrm{C}$ en invierno y de 28 a $28.8^{\circ} \mathrm{C}$ en verano (Secretaria de Marina 1985 citados en Pica \& Pineda 1999).

Debido a que la zona de estudio se localiza dentro del "área de afloramiento intenso" descrita por Merino (1992), el resultado esperado era observar en la temporada de tiburón ballena temperaturas más bajas, sin embargo el resultado fue inverso, lo cual pudiera deberse al nivel superficial al cual fue realizada la medición. Merino (1992), describe el agua de surgencia como una masa de agua que emerge desde los 220 a $250 \mathrm{~m}$ de profundidad a una velocidad cercana a los 10-2cm's-1 a lo largo de la cuesta este de la plataforma de Yucatán, adentrándose hasta la zona eufótica de la columna de agua que raramente rompe en la superficie. Por esta razón, para poder entender claramente el mecanismo de la surgencia en esta zona, son necesarios monitoreos que incluyan mediciones en toda la columna de agua.

La temperatura superficial del mar que prevaleció en la zona de estudio durante la temporada de tiburón ballena (entre 25 y $30^{\circ} \mathrm{C}$ ), se encuentra dentro del intervalo óptimo de temperatura reportado para la especie (Hoffman et al. 1981, Ketchum 2003, Graham et al. 2007; Sleeman et al. 2007, Nelson \& Eckert 2007).

La salinidad presentó variabilidad significativa entre temporadas (Fig. 3b) y, el hecho de que disminuya considerablemente en los meses de temporada de R. typus, probablemente se asocie al aporte de agua dulce que proviene de la alta precipitación que caracteriza estos meses (Riley \& Chester 1989, Millero 1996, HerreraSilveira et al. 1998, Duxbury et al. 2000). La alta salinidad en la zona ya ha sido previamente reportada en otros estudios llevados a cabo en la región, en donde se menciona que la causa de este máximo de salinidad es por la fricción de 


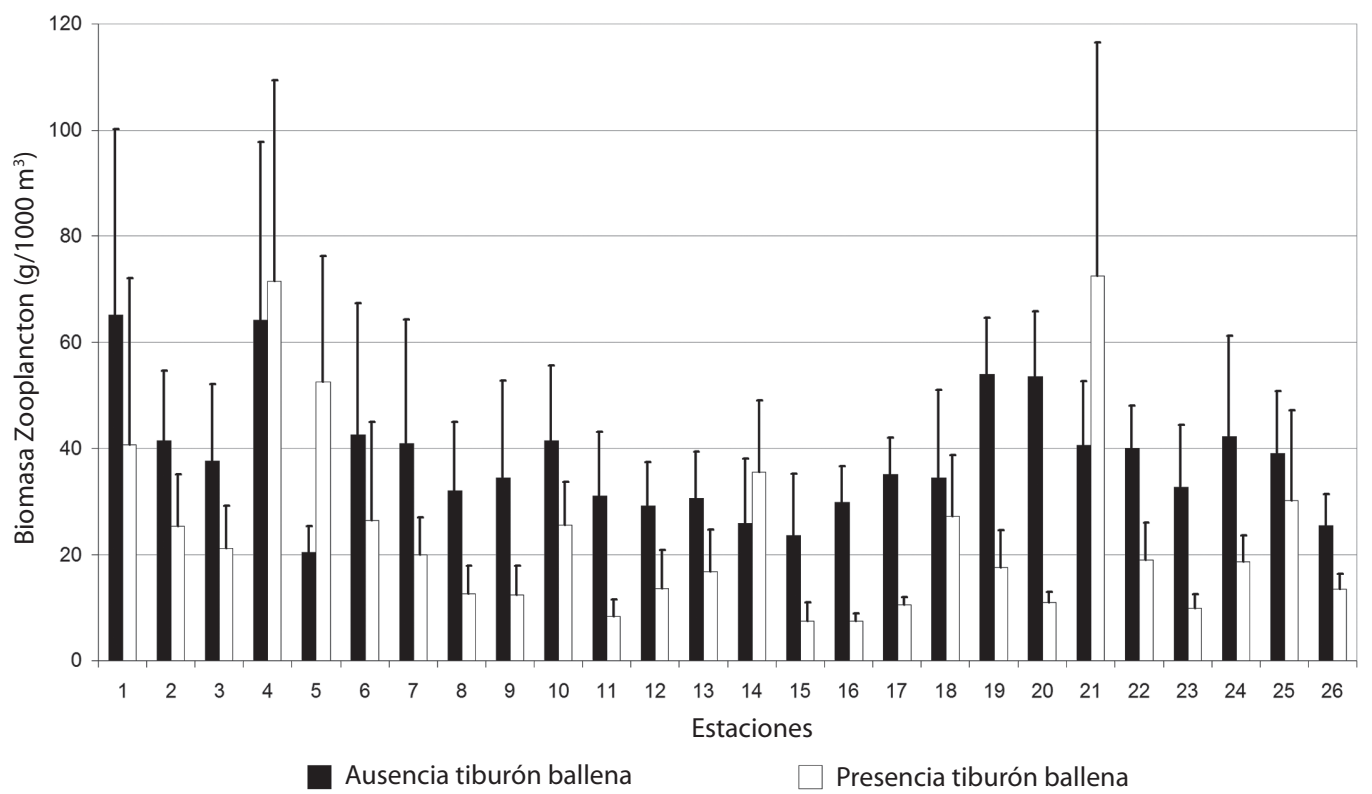

Fig. 5. Distribución espacio-temporal de la biomasa zooplanctónica promedio registrada durante 2005 y 2006 en cada estación de muestreo.

Fig 5. Spatial and temporal distribution of average zooplankton biomass recorded at each sample point, during 2005 and 2006.

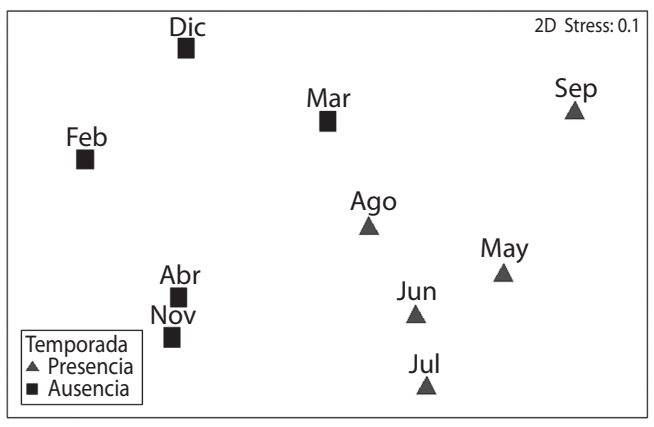

Fig. 6. Representación bidimensional del MDS para los meses de muestreo correspondientes a cada temporada (presencia y ausencia de tiburón ballena), de acuerdo a las variables ambientales monitoreadas.

Fig 6. Bidimensional diagram of the MDS analysis for the sampling months of each season (presence and absence of whale shark), according to environmental variables. las capas de la Corriente de Yucatán que toca la plataforma de la Península y aflora a la superficie levantando por encima de la plataforma el máximo de salinidad (36.3 a 36.5) característico de la masa de agua del Caribe denominada Agua Subtropical Intermedia (Cochrane 1972 citado en Ponce et al. 1999, Merino 1992). La salinidad reportada en el este estudio durante la temporada de tiburón ballena (34.7 y 36.9), es similar a la registrada en otros sitios del mundo en donde se congrega R. typus (Taylor \& Pearce 1999, Heyman et al. 2001, Ketchum 2003, Sleeman et al. 2007, Wilson et al. 2005).

Los valores de saturación de oxígeno registrados son similares a los ya reportados en la zona (Bogdanov 1969, Bessonov et al. 1971, De la Lanza et al. 1976, Merino 1992). La capa superficial del mar en temporada de ausencia 
CUADRO 1

Resultados SIMPER de variables ambientales para cada temporada

TABLE 1

SIMPER results of environmental variables of each season

\begin{tabular}{lcc}
\multicolumn{3}{c}{ Valor de disimilitud media entre temporadas (presencia y ausencia tiburón ballena): 24.40} \\
Coeficiente extinción luz (K1) & \% Contribución & 14.19 \\
Temperatura Superficial & 14.19 & 25.84 \\
Fosfatos $\left(\mathrm{PO}^{4}\right)$ & 11.65 & 36.86 \\
Biomasa Zooplanctónica & 11.02 & 47.81 \\
\% Saturación de oxígeno & 10.95 & 57.86 \\
Clorofila-a & 10.05 & 67.9 \\
Amonio $\left(\mathrm{NH}^{4}\right)$ & 10.04 & 77.84 \\
Nitratos $\left(\mathrm{NO}^{3}\right)$ & 9.94 & 85.24 \\
Silicatos $\left(\mathrm{SiO}^{4}\right)$ & 7.4 & 92.62 \\
Nitritos $\left(\mathrm{NO}^{2}\right)$ & 7.38 & 100
\end{tabular}

de tiburón ballena se encuentra cercana a la saturación (Fig. 3c) como tiende a estar en la mayoría del océano por el intercambio con la atmósfera (Riley \& Chester 1989, Millero 1996); mientras la capa superficial del agua en la temporada de presencia del tiburón ballena se encuentra sobresaturada de oxígeno (de 100 a 130 de porcentaje de saturación en la mayoría de los registros). Este resultado concuerda con lo observado por Merino (1983), quien deduce que la sobresaturación es consecuencia de la intensificación del fenómeno de surgencia durante primavera y verano. Dicha surgencia hace de la zona un punto de gran actividad biológica e incluso tomando en cuenta la respiración se alcanza sobresaturación de oxígeno en los primeros 30m (De La Lanza et al. 1976). Dentro del área de estudio no se observa un déficit de oxígeno por acumulación de materia orgánica.

El coeficiente de extinción de luz $\left(\mathrm{k}^{-1}\right)$, explica el grado de penetración de luz al ecosistema marino, un valor alto indica menor grado de penetración de luz traducido a mayor turbidez del agua (De La Lanza 1991). Esta variable presentó diferencias significativas entre temporadas, registrándose los valores más altos en temporada de presencia de tiburón ballena (Fig. 3d), lo cual pudiera estar relacionado con la mayor abundancia de organismos planctónicos. Cabe señalar, que en la zona no se observa mecanismos evidentes de resuspensión de sedimentos o descargas de efluentes cercanas.

En general, el comportamiento de los nutrientes en el Golfo y el Caribe Mexicano responden a los distintos eventos de circulación, mismos que están influenciados por la estacionalidad y fenómenos de afloramiento de aguas profundas, así como la confluencia de diferentes masas de agua, giros y hundimientos (De la Lanza 1991). El nitrógeno es uno de los micronutrientes limitantes en el medio marino y se reconoce que las aguas tanto del Golfo de México, como del Mar Caribe son pobres en nitratos, nitritos y amonio. Sin embargo, se sabe que para la zona las concentraciones de sus compuestos se modifican fuertemente en verano debido a la intensificación de la surgencia (Merino 1983). Durante la temporada de presencia de tiburón ballena, la concentración de clorofila- $a$ es mayor por el efecto fertilizador de la intensificación de la surgencia por lo que los compuestos de nitrógeno inorgánico están siendo consumidos en mayor medida para la síntesis biológica. A pesar de esto, la concentración del nitrógeno inorgánico total es mayor en temporada de presencia del tiburón (Fig. 3e). La concentración de fosfatos 
en la zona de estudio es relativamente alta en comparación a las reportadas para el Golfo de México y el Caribe (alrededor de $0.1 \mu \mathrm{mol} / 1$ ), presentándose las mayores concentraciones de este nutriente en temporada de ausencia de la especie cuando la surgencia es menos intensa (Fig. 3f), lo cual pudiera explicarse por el carácter cuasi-permanente de la surgencia (Merino 1992, Cereida-Estrada et al. 1998), los procesos de descomposición y resuspensión de materia orgánica en época de secas y la turbulencia producida durante el periodo de nortes.

El intervalo de concentraciones de silicatos $(1.2 \mu \mathrm{mol} / \mathrm{L}-37.2 \mu \mathrm{mol} / \mathrm{L})$ concuerda con lo reportado por Merino (1992). A pesar de que los silicatos son el nutriente menos estudiado en la zona, se ha reconocido que el agua de la corriente de Yucatán es pobre en dicho nutriente; sin embargo, se ha observado que para el Canal de Yucatán a profundidades mayores a los $200 \mathrm{~m}$ los silicatos incrementan considerablemente (Ponce et al. 1999), por lo que podrían estar potencialmente disponibles durante el afloramiento de aguas profundas característico de la zona. Las concentraciones de silicatos registradas entre temporadas no difirieron significativamente (Fig. 3g), sugiriendo que este nutriente no es limitante para la productividad en la zona de estudio.

Variables biológicas: El intervalo de concentración de $\mathrm{Cl}-a$ durante la temporada de tiburón fue mayor que el registrado en la temporada de ausencia de la especie (Fig. 4a), lo cual constituye un indicador del florecimiento de comunidades fitoplanctónicas debido a la variación estacional en la intensidad de la surgencia (Ruíz-Renteria \& Merino 1989, Merino 1992, Pérez de los Reyes et al. 1996). Los valores de $\mathrm{Cl}-a$ registrados se encuentran en el intervalo de valores observados previamente en la zona $\left(0.01\right.$ a $\left.5.9 \mathrm{mg} / \mathrm{m}^{3}\right)$ (Signoret et al. 1998 , Merino 1992).

Los patrones migratorios de las especies animales están asociados a la búsqueda de sitios de alimentación y/o sitios para su reproducción. En el caso del tiburón ballena, se ha observado que su distribución y abundancia depende de la disponibilidad del alimento, ya que los sitios de agregación estacional de la especie corresponden a zonas de alta productividad y por ende concentraciones masivas de plancton (Taylor 1996, Clark \& Nelson 1997, Colman 1997, Norman 2000, Taylor \& Pearce 1999, Eckert \& Stewart 2001, Wilson et al. 2001, Ketchum 2003, Graham et al., 2007).

Los estudios cuantitativos de disponibilidad de zooplancton en los sitios de agregación estacional del tiburón ballena son escasos. Para Bahía de La Paz (Baja California, México), durante los años 2001 y 2002 se reportaron máximos de biomasa zooplanctónica entre $413 \mathrm{ml} / 1000 \mathrm{~m} 3$ y $585 \mathrm{ml} / 1000 \mathrm{~m} 3$ con el método de volumen desplazado (Ketchum 2003). En este mismo trabajo, se reporta que en sitios con biomasa zooplanctónica menor a los $380 \mathrm{ml} / 1000 \mathrm{~m} 3$ no se reportó la presencia de tiburón ballena. Gunn et al. (1999) en el arrecife de Ningaloo en Australia, observó los picos de biomasa de zooplancton $(110 \mathrm{~g} / 1000 \mathrm{~m} 3$ $390 \mathrm{~g} / 1000 \mathrm{~m} 3$ ) en áreas donde se registraron las mayores abundancias de $R$. typus; al igual que en el presente trabajo.

Al comparar la biomasa zooplanctónica promedio entre temporadas (ausencia y presencia de tiburón ballena), los resultados no fueron los esperados, ya que la biomasa zooplanctónica en temporada de ausencia de la especie fue mayor (Fig. 4b). Algunas posibles causas que pudieran estar influenciando este resultado, son la mayor presión de pastoreo sobre la comunidad planctónica que ejerce la presencia del tiburón ballena, la distribución en parches de la biomasa zooplanctónica dentro del polígono de estudio y la variación en la composición zooplanctónica.

En estudios llevados a cabo por miembros del Mote Marine Laboratory de Florida (Estados Unidos) en esta zona, se ha calculado que el tiburón ballena consume alrededor de $28 \mathrm{Kg}$ de plancton al día (R. Hueter \& J. Timinsky 2009, com. pers.), lo cual refleja el claro efecto del pastoreo del tiburón ballena sobre las comunidades zooplanctónica. La distribución horizontal de biomasa zooplanctónica 
en parches, pudiera responder a la dinámica de corrientes que ha sido reportada en otros sitios de agregación de tiburón ballena (Clark \& Nelson 1997, Gunn et al. 1999, Ketchum 2003). La biomasa zooplanctónica registrada en el presente estudio, presentó una marcada variabilidad espacial, ya que algunas estaciones registraron acumulaciones masivas de zooplancton, en comparación con otros sitios que presentaron biomasas muy bajas (Fig. 5), lo cual robustece la hipótesis de que la variabilidad espacial de las biomasas de zooplancton se asocia con su distribución en parches.

En el trabajo realizado por CárdenasPalomo (2007), se reporta la variación en la composición de las muestras de zooplancton colectadas durante este estudio, registrándose como grupo dominante a los copépodos, sin embargo en los sitios de mayor abundancia de tiburón ballena la dominancia de este grupo fue menor, cobrando mayor importancia grupos como larvas de gasterópodos, huevos de pez, sergéstidos, quetognatos y apendicularias. Se sugiere en este mismo trabajo, una posible preferencia del tiburón ballena por el consumo de estos grupos, ya que son organismos de mayor talla en comparación con los copépodos y probablemente más ricos energéticamente.

La información presentada en el presente trabajo sugiere que la zona ubicada al norte del Caribe Mexicano es utilizada por el tiburón ballena principalmente como sitio de alimentación, ya que en los sitios donde fueron registradas las mayores abundancias de este pez, coincidieron con los máximos de biomasa zooplanctónica registrados. Adicionalmente, el 95\% de los tiburones avistados desde embarcación marina, se encontraba alimentándose y no fue registrada la presencia de crías en el área de estudio o algún evento de apareamiento. El análisis temporal de las variables ambientales analizadas permitió identificar que las condiciones hidrobiológicas son significativamente diferentes entre temporadas (presencia y ausencia de tiburón ballena), sugiriendo que la presencia de $R$. typus en la zona se asocia a las características del hábitat durante el periodo mayo-septiembre que favorecen la productividad biológica en la zona, por lo que se justifica ampliamente un programa de monitoreo durante este periodo del año y en series de tiempo más prolongadas.

\section{AGRADECIMIENTOS}

A los integrantes del laboratorio de productividad primaria y plancton del CINVESTAV-IPN Unidad Mérida, en especial a Nora Aguas, Uriel Ordoñez, Margarita Ornelas y Fany Merino. A los integrantes del proyecto DOMINO de la CONANP: Rafael de la Parra, Montserrat Trigo, Francisco Remolina y Jaime González. Este proyecto fue financiado por el Programa de Pequeñas Donaciones de PNUD.

\section{RESUMEN}

En la zona marina ubicada al norte del Caribe Mexicano se congregan grandes grupos de tiburón ballena (Rhincodon typus) entre mayo y septiembre de cada año. Se describe la variación espacio-temporal de la distribución del tiburón ballena al norte del Caribe mexicano y las condiciones fisicoquímicas y biológicas que prevalecen en la zona durante la temporada de presencia y ausencia de $R$. typus. De abril de 2005 a marzo de 2006, 26 estaciones de muestreo fueron monitoreadas registrando avistamientos de tiburón ballena y variables fisicoquímicas (temperatura, oxígeno disuelto, coeficiente de extinción de luz y salinidad) en 10 salidas de campo. En cada estación fueron recolectadas muestras zooplancton y de agua para determinación de clorofila- $a$ y nutrientes. Las condiciones fisicoquímicas fueron significativamente diferentes entre temporadas (ANOSIM, $R_{\text {global }}=0.632$ ). La temporada de presencia de $R$. typus estuvo caracterizada por valores bajos de salinidad, mayores valores de temperatura y oxígeno disuelto; mayores de nitrógeno inorgánico disuelto y concentraciones de clorofila- $a$ y aunque la biomasa promedio de zooplancton fue menor que en la temporada de ausencia de $R$. typus, los valores máximos de biomasa zooplanctónica se registraron en el periodo de presencia y en sitios donde se registraron las mayores abundancias de la especie, apoyando la hipótesis de que el sitio es utilizado por la especie principalmente como zona de alimentación. Las condiciones físicoquímicas de la zona de estudio que favorecen la productividad biológica explican la variabilidad espacial y temporal de $R$. typus al norte del Caribe Mexicano.

Palabras clave: Rhincodon typus, surgencia, hábitat, patrón de distribución, Caribe Mexicano 


\section{REFERENCIAS}

Arriaga-Cabrera, L., V. Aguilar-Sierra \& J.M. Espinoza. 2009. Regiones prioritarias y planeación para la conservación de la biodiversidad, p. 433-457. In Conabio (ed.). Capital natural de México. Vol. II: Estado de conservación y tendencias de cambio. Conabio, México.

Bessonov, N., O. González \& A. Elizarov. 1971. Resultados de las investigaciones cubano-soviéticas en el Banco de Campeche. Departamento de Hidroquímica del C.I.P., Cuba.

Bogdanov, D.V. 1969. Some oceanographic features of the Gulf of Mexico and the Caribbean Sea. Sovietic Cuban Fishery Research U.S. Department of Comerse Spring Geld. Jerusalem: Israel Program for Scientific Translations, Jerusalen, Israel.

Cárdenas-Palomo N. 2007. Distribución espacio-temporal de variables hidrobiológicas asociadas con el uso del hábitat del tiburón ballena (Rhincodon typus) en el noreste de la Península de Yucatán. Tesis de Maestría, Instituto Politécnico Nacional, Unidad Mérida, Yucatán, México.

Cárdenas-Tórres, N., R. Enríquez \& N. Rodíguez -Dowdell. 2007. Community-based management through ecoturism in Bahía de los Angeles, México. Fish. Res. 84: 114-118.

Cereida-Estrada, S., N. Melo-González, R. Pérez de los Reyes, I.R. Victoria \& F.E. Müller-Karger. 1998. La densidad de pigmentos fotosintéticos, un indicador del carácter e intensidad de los procesos oceanográficos en el occidente del gran Caribe. Boletín SOMETCUBA 4: 1-13.

Clark, E. \& D.R. Nelson. 1997. Young whale sharks, Rhincodon typus, feeding on a copepod bloom near La Paz, Mexico. Environ. Biol. Fishes. 50: 63-73.

Clarke, K.R. \& R.M. Warwick. 1994. Change in Marine Communities: An Approach to Statistical Analysis and Interpretation. Plymouth Marine Laboratory, Plymouth, Reino Unido.

Cochrane, J.D. 1966. The Yucatan Current, upwelling off Northeastern Yucatan, and currents and waters of Western Equatorial Atlantic. Oceanography of the Gulf of Mexico. Progress Rep. Universidad de Texas, USA. Ref. No. 66-23T, p. 14-32.

Colman, J.G. 1997. A review of the biology and ecology of the Whale Shark. J. Fish Biol. 51: 1219-1234.
Compagno, L.J. 1984. FAO Species catalogue. Vol. 4. Sharks of the world. Part. 1 FAO Fisheries Synopsis 25: 209-211.

De La Lanza, E.G., J.E. Rodríguez \& S. Guevara. 1976. Hidrología de la Bahía de Campeche y Norte de Yucatán. Memorias I Reunión Latinoamericana de Ciencia y Tecnología oceanográfica México. 2: 108161.

De la Lanza, E.G. 1991. Oceanografía de mares mexicanos. AGT S.A., México D.F., México.

Duffy, A.J. 2002. Distribution, seasonality, lengths, and feeding behaviour of whale sharks (Rhincodon typus) observed in New Zealand waters. New Zeal J. Mar. Fresh. 36: 565-570.

Duxbury, A.C., A.B. Duxbury \& K.A. Sverdrup. 2000. An Introduction to the World's Oceans. Mc Graw-Hill Companies, Boston, EEUU.

Eckert, S.A. \& B.S. Stewart. 2001. Telemetry and Satellite Tracking of Whale Sharks Rhincodon typus, in the Sea of Cortez, Mexico, and the North Pacific Ocean. Environ. Biol. Fishes. 60: 299-308.

García, C. 1990. Influencia del campo de presiones en la circulación del estrecho de Yucatán. Chapman Conference on the Physics of the Gulf of Mexico, junio 1989, St. Petesburg, Florida, 14 p.

Graham, R.T., C.M. Roberts \& J. Smart. 2007. Patterns of diving whale shark over variable time scales and in relation to a predictable food source, p. 73. In T.R. Irvine \& J.K. Keesing (eds.). The First International Whale shark conference: promoting international collaboration in whale shark conservation, science and management. Conference Overview, abstracts and supplementary proceedings. CSIRO Marine and atmospheric research. Perth, Australia.

Gunn, J.S., J.D. Stevens, T.L. Davis \& B.M. Norman. 1999. Observations on the Short-Term Movements and Behavior of the Whale Sharks (Rhincodon typus) at Ningaloo Reef, Western Australia. Mar. Biol. 135: 553-559.

Herrera-Silveira, J., J. Ramírez \& A. Zaldivar. 1998. Overview and characterization of the hydrology and primary producer communities of selected coastal lagoons of Yucatan, México. Aquat. Ecosyst. Health and Manag. 1: 353-372.

Heyman, W.D., R.T. Graham, B. Kjerfve \& R.E. Johannes. 2001. Whale sharks Rhincodon typus aggregate to feed on fish spawn in Belize. Mar. Ecol. Prog. Ser. 215: 275-282. 
Hoffman, W., T.H. Fritts \& R.P. Reynolds. 1981. Whale shark associated with fish schools off south Texas. North-East Gulf Sciences 5: 55-57.

Ketchum, J.T. 2003. Distribución espacio temporal y ecología alimentaria del tiburón ballena (Rhincodon typus) en la Bahía de la Paz y Zonas adyacentes en el suroeste del Golfo de California. Tesis de Maestría, Instituto Politecnico Nacional, La Paz Baja California Sur, México.

Merino, I.M. 1983. Estudios ambientales del Arrecife de Puerto Morelos, Quintana Roo. Informe del proyecto de Investigación PAMOR. UACP y P-CCH. Maestría en Ciencias del Mar, Universidad Nacional Autónoma de México, México D.F., México.

Merino, I.M. 1992. Afloramiento en la Plataforma de Yucatán. Estructura y Fertilización. Tesis de Doctorado, Universidad Nacional Autónoma de México, México D.F., México.

Millero, F.J. 1996. Chemical Oceanography. CRC Marine Science Series. 531 p. CRC, Boca Raton, Florida, EEUU.

Nelson, J.D. \& S.A. Eckert. 2007. Foraging Ecology by whale sharks (Rhincodon typus) within Bahía de los Angeles, Baja California Norte, México. Fish. Res. 84: 47-64.

Norman, B.M. 2000. IUCN Red List of Threatened Species. IUCN, Gland, Switzerland and Cambridge, Inglaterra.

Omori, M. \& T. Ikeda. 1984. Methods in Marine Zooplankton Ecology. J. Wiley \& Sons, Nueva York, EEUU.

Parsons, T., Y. Maita \& C. Lally. 1984. A Manual of Chemical and Biological Methods of Seawater Analysis. Pergamon Press, Oxford, Inglaterra.

Pérez de los Reyes, R., V.I. Del Río, P.M. Signoret, G.A. Gallegos, R.I. Penié, V.C. Gil, C. Bulit, N. MeloGonzález, F. Müller-Karger, S. Cerdeira Estrada \& M. Merino-Ibarra. 1996. Reporte de un frente hidrológico al noreste de la Península de Yucatán. Mani-festaciones químicas e hidrobiológicas. Bol. Soc. Meteorol. Cuba 2: 1-16 (también disponible en línea: http://www.xoc.uam.mx/uam/divisiones/cbs/ reporte.html).

Pica, G.Y. \& R. Pineda. 1999. Golfo de México y Mar Caribe. Oceanografía física, p. 31-78. In G. De La Lanza (ed.). Oceanografía de mares mexicanos. AGT, México D.F., México.
Ponce, V.M., L.R. Pineda \& G. Pica. 1999. Golfo de México y Mar Caribe. Oceanografía Química, p. 79-115. In G. De La Lanza (ed.). Oceanografía de mares mexicanos. AGT, México D.F., México.

Quiros, A. 2007. Tourist compliance to a code of conduct and the resulting effects on whale shark (Rhincodon typus) behavior in Donsol, Philippines. Fish. Res. 84: 102-108

Reyes-Mendoza, O. \& N. Cárdenas-Palomo. 2006. Distribución del tiburón ballena en el norte del Caribe Mexicano. Reporte del estudio técnico del programa PRODERS. Quintana Roo, México. 20 p.

Riley, J.P. \& R. Chester. 1989. Introducción a la Química Marina. AGT, México D.F., México.

Ruíz-Renteria \& M. Merino. 1989. Upwelling off the north coast of the Yucatan Peninsula, p. 149-160. In E.A. Chávez (ed.). Memorias del Seminario México-Australia sobre Ciencias Marinas. Yucatán y Quintana Roo, México.

SEMARNAT \& CONANP. 2005. Estudio Previo Justificativo para el Establecimiento del Área Natural Protegida. Reserva de la Biosfera del Tiburón Ballena. Quintana Roo, México.

Signoret, M.C., C. Built \& R. Pérez de los Reyes. 1998. Patrones de distribución de clorofila-a y producción primaria en aguas del Golfo de México y Mar de Cortéz. Hidrobiológica 8: 81-88.

Sleeman, J., M. Meekan, C. Bradshaw \& C. Steinberg. 2007. The influence of oceanographic and atmospheric processes on whale shark abundance at Ningaloo Reef, Western Australia, p. 90. In T.R. Irvine \& J.K. Keesing (eds.). The First International Whale shark conference: promoting international collaboration in whale shark conservation, science and management. Conference Overview, abstracts and supplementary proceedings. CSIRO Marine and atmospheric research. Perth, Australia.

Solares, I. 2005. Evaluación socioeconómica rápida de las comunidades del interior y la zona de influencia del APFF Yum balam: una herramienta para definir estrategias de conservación para el desarrollo, dentro de una región prioritaria para la conservación. Cap. III Holbox. Reporte Final de proyecto. CONANP. Quintana Roo, México. p. 213-261.

Strickland, J.D. \& T.R. Parsons. 1972. A Practical Handbook of Seawater Analysis. Bull. Fish. Res. Board Can. 67: 207-211.

Taylor, J.G. 1996. Seasonal occurrence, distribution and movements of the Whale Shark, Rhincodon typus, at 
Ningaloo Reef, Western Australia. Mar. Freshw. Res. 47: $637-42$.

Taylor, J.G. \& A.F. Pearce. 1999. Ningaloo Reef currents: implications for coral spawn dispersal, zooplankton and whale shark abundance. J. R. Soc. West. Aust. 82: 57-65.

UNESCO. 1979. Zooplankton Sampling. Monographs on oceanographic methodology. UNESCO, France.

Wilson, S.G., J.G. Taylor \& A.F. Pearce. 2001. The seasonal aggregation of whale sharks at Ningaloo Reef, Western Australia: currents, migrations and the El Niño/Southern Oscillation. Environ. Biol. Fish. 61: 1-11.
Wilson, S.G., J.J. Polovina, B.S. Stewart \& M. Meekan. 2005. Movements of whale sharks (Rhincodon typus) tagged at Ningaloo Reef, Western Australia. Mar. Biol. 148: 1157-1166.

\section{REFERENCIA DE INTERNET}

DOMINO, 2005. Proyecto Domino: Proyecto del tiburón ballena del Atlántico mexicano. Resultados 2005. Sobre vuelos realizados con fechas 24/06/2005, 13/07/2005, 15/06/2005, 05/08/2005, 16/08/2005, 09/09/2005, 31/05/2005 por R. de la Parra Venegas, M. Trigo-Mendoza, F. Remolina-Suárez, J. GonzálezCano, D. Ramírez-Macias y H. Linnestad (Consultado: 1 Setiembre 2008, http://www.domino.conanp. gob.mx/reul2005.htm). 